\title{
On an equation in cyclotomic numbers
}

\author{
by \\ Roberto Dvornicich (Pisa)
}

1. Introduction. Let $K$ be a field of characteristic 0 and let $a, b, c$ be non-zero elements of $K$. In connection with the problem of studying the possible degree of $(f(x), g(x))$, where $f(x)=x^{n}+A x^{m}+B$ is a trinomial and $g(x)=x^{Q}-C$ is a binomial, A. Schinzel asked (oral communication) the following

QUESTION. Let $\zeta_{1}, \zeta_{2}$ be two complex roots of unity with least common $\operatorname{order} Q$ and suppose that $m, n$ are integers such that $(m, n, Q)=1$ and

$$
\begin{aligned}
\left|\zeta_{1}^{m}-1\right|^{m}\left|\zeta_{1}^{n-m}-1\right|^{n-m}\left|\zeta_{1}^{n}-1\right|^{-n} & \\
& =\left|\zeta_{2}^{m}-1\right|^{m}\left|\zeta_{2}^{n-m}-1\right|^{n-m}\left|\zeta_{2}^{n}-1\right|^{-n},
\end{aligned}
$$

where none of the six absolute values is 0 . Is it true that then $\zeta_{2}=\zeta_{1}^{ \pm 1}$ ?

For the connection between these two problems, see Schinzel [4].

We remark that there is a symmetry among the numbers $m, n-m,-n$ : in fact, $\left|\zeta^{n}-1\right|=\left|\zeta^{-n}-1\right|$ and we can rewrite equation (1) in the symmetric form

$$
\left|\zeta_{1}^{a}-1\right|^{a}\left|\zeta_{1}^{b}-1\right|^{b}\left|\zeta_{1}^{c}-1\right|^{c}=\left|\zeta_{2}^{a}-1\right|^{a}\left|\zeta_{2}^{b}-1\right|^{b}\left|\zeta_{2}^{c}-1\right|^{c},
$$

where $\{a, b, c\}=\{m, n-m,-n\}$. Assuming again that none of the six absolute values is zero, the hypotheses in Schinzel's question can then be translated into $a+b+c=0$ and $(a, b, c, Q)=1$.

The aim of this paper is to prove that Schinzel's question has a positive answer with essentially one exception. More precisely, we shall prove

THEOREM 1. Let $\zeta_{1}, \zeta_{2}, Q, m, n$ be as above and assume that (1) holds. Then either $\zeta_{2}=\zeta_{1}^{ \pm 1}$ or $Q=10,\{m, n-m,-n\}=\{x, 3 x,-4 x\}$ for some integer $x$ with $(x, 10)=1$, and $\zeta_{1}, \zeta_{2}$ are any two primitive tenth roots of unity. 
Let $\zeta=\zeta_{Q}$ be a primitive $Q$ th root of unity. According to a definition given by Conrad [1], the multiplicative group generated by the numbers $\zeta^{\nu}-1$ with $\nu \not \equiv 0(\bmod Q)$ modulo roots of unity is the group $D^{(Q)}$ of cyclotomic numbers. This group and its subgroups, in particular the subgroup of cyclotomic units, have been studied by many authors (see for instance [3], [6]-[8]). A classification of short multiplicative relations among cyclotomic numbers, however, is not available in the literature, and even for a simple equation like (1) there is no automatic way to find all solutions.

The main tool for the proof of Theorem 1 will be a result by Ennola [3], which gives necessary and sufficient conditions for a relation among cyclotomic numbers to hold. In Section 2 we shall recall Ennola's result and we shall examine a few small values of $Q$. After some preliminary lemmas (Section 3), we shall split our analysis into a fairly large number of cases. The proof of Theorem 1 will result from the combination of Propositions $1-10$ of Sections 4 and 5 . As the proof is rather technical, we shall leave enough detail in the general arguments, but we shall omit completely the verifications when these concern a finite number of cases, which can very easily be checked by a computer search.

2. Ennola's result and a few small values of $Q$. We shall always denote by $\zeta$ a primitive $Q$ th root of unity. We briefly recall the main result of Ennola [3].

For $x \in \mathbb{Z} / Q \mathbb{Z}$, let

$$
A_{x}=\log \left|\zeta^{x}-1\right|,
$$

and consider a linear combination with integer coefficients of the $A_{x}$,

$$
R=\sum_{x=1}^{Q-1} C_{x} A_{x}
$$

For an even character $\chi$ mod $Q$ of conductor $f>1$ and for each $d$ such that $f|d| Q$, we define

$$
T(\chi, d, R)=\sum_{\substack{x=1 \\(x, d)=1}}^{d-1} \chi(x) C_{(Q / d) x}
$$

and

$$
Y(\chi, R)=\sum_{\substack{d \\ f|d| Q}} \frac{1}{\phi(d)} \prod_{p \mid d}(1-\bar{\chi}(p)) T(\chi, d, R) ;
$$

moreover, for all prime numbers $p \mid Q$ we define 


$$
Y_{p}(R)=\sum_{x=1}^{p^{\gamma_{p}}-1}\left(x, p^{\gamma_{p}}\right) C_{\left(Q / p^{\gamma_{p}}\right) x},
$$

where $p^{\gamma_{p}} \| Q$. Then we have the following

Theorem 2 (Ennola). We have $R=0$ if and only if

$$
Y(\chi, R)=0 \quad \text { for every even character } \chi \neq \chi_{1}
$$

and

$$
Y_{p}(R)=0 \quad \text { for every prime } p \text { dividing } m .
$$

Throughout the paper we shall keep the notation of (1)-(6), which is borrowed from [3] with the only exception that in the present paper the common order of $\zeta_{1}$ and $\zeta_{2}$ is called $Q$. Moreover, for a positive integer $d$ we shall denote by $\mu_{d}$ the group of complex $d$ th roots of unity and by $\zeta_{d}$ (for $d>2$ ) a primitive $d$ th root of unity. The symbol $\zeta$ without a subscript will always stand for a primitive $Q$ th root of unity.

Let $\zeta_{1}=\zeta^{l}, \zeta_{2}=\zeta^{k}$. In Ennola's notation, relation (1) reads as $R=0$ where

$$
\begin{aligned}
R= & m A_{l m}+(n-m) A_{l(n-m)}-n A_{l n} \\
& -\left(m A_{k m}+(n-m) A_{k(n-m)}-n A_{k n}\right) .
\end{aligned}
$$

We shall derive most of our results as consequences of relations (7). For $Q \in\{1,2,3,4,6\}$, however, there is no even character $\chi \neq \chi_{1}$ modulo $Q$, so conditions (7) are empty. We leave it to the reader to verify that equations (8) are sufficient to prove Theorem 1 for these particular values of $Q$.

Therefore, we shall assume $Q \notin\{1,2,3,4,6\}$ from now on. Moreover, we shall deal separately with the case when $Q \equiv 2(\bmod 4)$, since in this case there is no primitive character with modulus $Q$.

3. Preliminary results. We recall without proof the following

Lemma 1. Let $C$ be the maximal conductor of an even primitive character modulo a divisor of $Q$. Then

$$
C=\left\{\begin{array}{ll}
Q^{\prime} & \text { if } Q=2 Q^{\prime} \\
Q & \text { otherwise }
\end{array} \text { and } Q^{\prime}\right. \text { is odd }
$$

A primitive character mod $C$ is also a Dirichlet character mod $C$. Let $G$ be the group of all even Dirichlet characters $\bmod C$ and let $K$ be the subgroup of $G$ generated by the set $X$ of primitive even characters, i.e. of even Dirichlet characters of maximal conductor.

Lemma 2. $K=G$ unless $Q=2^{a} \cdot 3, a \geq 3$, in which case $[G: K]=2$.

Proof. We split the proof in a number of cases. 
CAsE 1: $Q$ is odd or $Q=2^{a}$. This case is trivial if $Q$ is a prime power, since the group of even Dirichlet characters is cyclic and any generator must clearly have maximal conductor. Let then $Q=q_{1} \ldots q_{r}=p_{1}^{a_{1}} \ldots p_{r}^{a_{r}}$ be the factorization of $Q$ into primes, $2<p_{1}<\ldots<p_{r}, r \geq 2$ and let $\chi_{1}, \ldots, \chi_{r}$ be Dirichlet characters induced by (odd) generators of the primitive characters $\bmod q_{1}, \ldots, q_{r}$, respectively. Note that $\chi_{i}^{2}$ also has conductor $q_{i}$ unless $q_{i}=3$, so in particular $\chi_{i}^{2}$ has conductor $q_{i}$ for $i \geq 2$. We have

$$
\left(\chi_{1} \chi_{2} \chi_{3}^{2} \ldots \chi_{r}^{2}\right) \cdot\left(\chi_{1} \chi_{2}^{-1} \chi_{3}^{-2} \ldots \chi_{r}^{-2}\right)=\chi_{1}^{2} \in K
$$

and, similarly, $\chi_{i}^{2} \in K$ for all $i=1, \ldots, r$. Also,

$$
\left(\chi_{1} \chi_{2} \chi_{3}^{2} \ldots \chi_{r}^{2}\right) \cdot\left(\chi_{3}^{-2} \ldots \chi_{r}^{-2}\right)=\chi_{1} \chi_{2} \in K
$$

and, similarly, $\chi_{1} \chi_{i} \in K$ for all $i>1$. Finally, if $1<i<j$ we have $\chi_{1}^{-2} \cdot \chi_{1} \chi_{i} \cdot \chi_{1} \chi_{j}=\chi_{i} \chi_{j} \in K$.

The case follows since $G$ is generated by the set $\left\{\chi_{i} \chi_{j} \mid 1 \leq i \leq j \leq r\right\}$.

CAsE 2: $Q=2 Q^{\prime}$ and $Q^{\prime}$ odd. By Lemma 1, we have to consider the characters $\bmod Q^{\prime}$, so Case 1 applies.

CASE 3: $Q=2^{a} Q^{\prime}, a \geq 2, Q^{\prime}$ odd and $Q^{\prime}>3$. Let $Q^{\prime}=q_{1} \ldots q_{r}=$ $p_{1}^{a_{1}} \ldots p_{r}^{a_{r}}$. The assumption $Q^{\prime}>3$ implies that either $r \geq 2$ or $r=1$ and $q_{1}>3$.

Let $\chi_{0}$ be a generator of the even characters mod $2^{a}$ and let $\chi^{(4)}$ be the (odd) primitive character mod 4. As in Case 1, we see immediately that $\chi_{i}^{2} \in K$ and $\chi_{i} \chi_{j} \in K$ for $0 \leq i \leq j \leq r$. Also, $\chi_{0} \in K$, since for $a=2$ we have $\chi_{0}=1$ and, for $a>2$ :

- if $r \geq 2$, then $\chi_{0} \chi_{1} \chi_{2} \chi_{3}^{2} \cdots \chi_{r}^{2} \in K$ implies that $\chi_{0} \in K$;

- if $r=1$, then $\chi_{0} \chi_{1}^{2} \in K$ implies that $\chi_{0} \in K$.

Furthermore,

$$
\chi^{(4)} \chi_{0} \chi_{1} \chi_{2}^{2} \ldots \chi_{r}^{2} \in K \Rightarrow \chi^{(4)} \chi_{1} \in K
$$

and $\chi^{(4)} \chi_{i}=\chi^{(4)} \chi_{1} \cdot \chi_{1} \chi_{i} \cdot \chi_{1}^{-2} \in K$ for all $i \geq 1$. Since all odd characters $\bmod 2^{a}$ are of the form $\chi_{0}^{b} \chi^{(4)}$ and $\chi_{0}^{b} \chi^{(4)} \chi_{i} \in K$ for all $i \geq 1$, it follows that $K$ is again the full group of even characters.

CASE 4: $Q=2^{a} \cdot 3, a \geq 2$. If $a=2$, then $\chi^{(4)} \chi_{1} \in K$ and generates the group of even characters mod 12. If $a>2$, all characters of maximal conductor are contained in the subgroup generated by $\chi^{(4)} \chi_{0} \chi_{1}$, which has order $2^{a-2}$, while $G$ has order $2^{a-1}$.

COROLlary 1. Let $k$ be such that $\chi(k)=1$ for all even primitive characters of maximal conductor $C$. Then

(a) $k \equiv \pm 1, \pm i(\bmod Q)$ if $Q=2^{a} \cdot 3, a \geq 3$, where $i$ is defined by the congruences $i \equiv 2^{a-1}+1\left(\bmod 2^{a}\right), i \equiv-1(\bmod 3)$, 
(b) $k \equiv \pm 1\left(\bmod Q^{\prime}\right)$ if $Q=2 Q^{\prime}, Q^{\prime}$ odd;

(c) $k \equiv \pm 1(\bmod Q)$ otherwise.

Proof. The case $Q=2^{a} \cdot 3$ is established by looking at the kernel of $\chi^{(4)} \chi_{0} \chi_{1}$. The remaining cases are direct consequences of Cases $1-3$ of Lemma 2.

4. The case when at least one root is primitive. Throughout this section we shall assume that either $\zeta_{1}$ or $\zeta_{2}$ is a primitive $Q$ th root of unity. Without loss of generality, we shall also assume that $\zeta_{1}=\zeta$.

4.1. The case $Q \not \equiv 2(\bmod 4)$. If $Q$ is odd, we can improve on Lemma 2 as follows. Let $Q=q_{1} \ldots q_{r}=p_{1}^{a_{1}} \ldots p_{r}^{a_{r}}$ be the prime factorization of $Q$, $3 \leq p_{1}<\ldots<p_{r}, r \geq 2$. Let $\chi_{1}, \ldots, \chi_{r}$ be as in Lemma 2. Any Dirichlet character $\bmod Q$ can be written in the form $\chi=\chi_{1}^{i_{1}} \ldots \chi_{r}^{i_{r}}$, and the subgroup $G$ of even Dirichlet characters is defined by the equation $i_{1}+\ldots+i_{r} \equiv 0$ $(\bmod 2)$. The following lemma is very similar in spirit to the results of $[5$, Sect. 3].

Lemma 3. If $Q>3$ is odd, the set $X$ of Dirichlet characters of maximal conductor $Q$ is not contained in the union of two maximal subgroups $H_{0}, K_{0}$ of $G$ unless $Q$ can be written as $Q=3 Q_{1} Q_{2}$ with $Q_{1}, Q_{2}>1,\left(3, Q_{1}\right)=$ $\left(3, Q_{2}\right)=\left(Q_{1}, Q_{2}\right)=1$ and the subgroups $H_{0}, K_{0}$ are defined by the equations

$$
\sum_{\substack{h \\ p_{h} \mid Q_{1}}} i_{h} \equiv 0(\bmod 2), \quad \sum_{\substack{h \\ p_{h} \mid Q_{2}}} i_{h} \equiv 0(\bmod 2)
$$

respectively.

Proof. The conclusion is trivial if $G$ is cyclic, since in this case any generator of $G$ has maximal conductor and cannot be contained in any proper subgroup. Hence from now on we can suppose that the number $r$ of distinct prime factors of $Q$ is $\geq 2$.

We recall that any maximal subgroup of a finite abelian group has prime index in the full group.

CASE 1: $\left[G: H_{0}\right]=\ell_{1},\left[G: K_{0}\right]=\ell_{2}, \ell_{1}, \ell_{2}$ primes $>2$. The equations defining $H_{0}$ and $K_{0}$ must be of the form

$$
\begin{aligned}
a_{1} i_{1}+\ldots+a_{r} i_{r} & \equiv 0\left(\bmod \ell_{1}\right), \\
b_{1} i_{1}+\ldots+b_{r} i_{r} & \equiv 0\left(\bmod \ell_{2}\right) .
\end{aligned}
$$

REMARK 1. If $a_{i} \not \equiv 0\left(\bmod \ell_{1}\right)\left(\operatorname{resp} . b_{i} \not \equiv 0\left(\bmod \ell_{2}\right)\right)$ then necessarily $p_{i}=\ell_{1}$ and $q_{i} \geq \ell_{1}^{2}$ or $p_{i} \equiv 1\left(\bmod \ell_{1}\right)\left(\operatorname{resp} . p_{i}=\ell_{2}\right.$ and $q_{i} \geq \ell_{2}^{2}$ or $p_{i} \equiv 1$ $\left.\left(\bmod \ell_{2}\right)\right)$. In particular, $a_{i} \equiv 0\left(\bmod \ell_{1}\right)$ and $b_{i} \equiv 0\left(\bmod \ell_{2}\right)$ if $q_{i}=3$ or $q_{i}=5$. 
If there exists a prime $p_{h}>3$ such that $a_{h} \not \equiv 0\left(\bmod \ell_{1}\right)$ and $b_{h} \not \equiv 0$ $\left(\bmod \ell_{2}\right)$, let $j=1$ if $p_{1}=3$ and $j$ be any index such that $j \neq h$ otherwise. Then one of the even $r$-tuples $\left(i_{1}, \ldots, i_{r}\right)$ defined by

$$
i_{h}=1,-1,3, i_{j}=1, i_{\lambda}=2 \text { for } \lambda \neq h, j
$$

does not satisfy either of equations (10) and (11). (In what follows, we say that an even $r$-tuple is good if it does not satisfy either of the equations defining the subgroups $H_{0}$ and $K_{0}$.)

If there exist two distinct primes $p_{h}, p_{j}>3$ such that $a_{h} \not \equiv 0\left(\bmod \ell_{1}\right)$ and $b_{j} \not \equiv 0\left(\bmod \ell_{2}\right)$, suppose, by symmetry, that $p_{h}<p_{j}$. Then one of the $r$-tuples $\left(i_{1}, \ldots, i_{r}\right)$ defined by

$$
\begin{cases}i_{h}= \pm 1, i_{j}= \pm 1 \text { and } i_{\lambda}=2 \text { for } \lambda \neq h, j & \text { if } p_{1}>3 \\ i_{h}= \pm 1, i_{j}= \pm 2, i_{1}=1, i_{\lambda}=2 \text { for } \lambda \neq 1, h, j & \text { otherwise }\end{cases}
$$

is good. So, since neither of equations (10) and (11) can be empty, we are left with the case (up to symmetry between $\ell_{1}$ and $\ell_{2}$ ) when $p_{1}=3, \ell_{2}=3$ and

$$
a_{1} i_{1}+\ldots+a_{r} i_{r} \equiv 0\left(\bmod \ell_{1}\right), \quad i_{1} \equiv 0(\bmod 3) .
$$

If $a_{h} \equiv 0\left(\bmod \ell_{1}\right)$ for all $h>1$, then the $r$-tuple defined by $i_{1}=i_{2}=1$, $i_{\lambda}=2$ for $\lambda>2$ is good. If $a_{h} \not \equiv 0\left(\bmod \ell_{1}\right)$ for some $h>1$, then at least one of the $r$-tuples defined by $i_{1}=1, i_{h}= \pm 1, i_{\lambda}=2$ for $\lambda \neq 1, h$ is good.

CASE $2:\left[G: H_{0}\right]=2$ and $\left[G: K_{0}\right]=\ell>2$. The group $G / 2 G$ has order $2^{r}$ if all $p_{i}$ are congruent to $1 \bmod 4$, and $2^{r-1}$ otherwise. In any case $G$ has $2^{r-1}-1$ subgroups of index 2 given by equations of type

$$
\sum_{h \in S} i_{h} \equiv 0(\bmod 2), \quad S \neq \emptyset,\{1, \ldots, r\}
$$

(the interchange between $S$ and its complementary set $\{1, \ldots, r\} \backslash S$ gives the same subgroup). These are all subgroups of index 2 if there exists at least one prime $p_{h}$ which is congruent to $3 \bmod 4$. If $p_{h} \equiv 1(\bmod 4)$ for all $i$, then we also have the $2^{r-1}$ subgroups given by equations of type

$$
\varepsilon_{1} i_{1}+\ldots+\varepsilon_{r} i_{r} \equiv 0(\bmod 4),
$$

where $\varepsilon_{h}= \pm 1$ and changing all signs gives rise to the same subgroup. So this case splits into two subcases.

SuBCASE 2a: The relevant equations are:

$$
\begin{gathered}
\varepsilon_{1} i_{1}+\ldots+\varepsilon_{r} i_{r} \equiv 0(\bmod 4), \quad \varepsilon_{h}= \pm 1, \\
a_{1} i_{1}+\ldots+a_{r} i_{r} \equiv 0(\bmod \ell),
\end{gathered}
$$

and $p_{h} \equiv 1(\bmod 4)$ for all $h$. 
Choose an index $h$ such that $a_{h} \not \equiv 0(\bmod \ell)$, and let $j \neq h$ be any other index. Then at least one of the $r$-tuples

$$
i_{h}= \pm 1, \pm 3, i_{j}=1, i_{\lambda}=2 \text { for } \lambda \neq h, j
$$

is good. (Note that $\chi_{h}^{ \pm 1} \neq \chi_{h}^{\mp 3}$ by Remark 1.)

SubCASE $2 b$ : The relevant equations are:

$$
\begin{gathered}
\sum_{h \in S} i_{h} \equiv 0(\bmod 2), \quad S \neq \emptyset,\{1, \ldots, r\}, \\
a_{1} i_{1}+\ldots+a_{r} i_{r} \equiv 0(\bmod \ell) .
\end{gathered}
$$

Note that, by Remark 1 , if $q_{i}=3,5$ then $a_{i}=0$. Interchanging $S$ with its complementary set if necessary, we may suppose that there exists an index $h \notin S$ such that $a_{h} \not \equiv 0(\bmod \ell)$. Let $j=1$ if $1 \in S$ and $j$ be any index such that $j \in S$ otherwise. Then at least one of the two $r$-tuples

$$
\begin{cases}i_{1}=1, i_{h}= \pm 1, i_{\lambda}=2 \text { for } \lambda \neq 1, h & \text { if } 1 \in S \\ i_{1}=1, i_{j}=1, i_{h}= \pm 2, i_{\lambda}=2 \text { for } \lambda \neq 1, h, j & \text { otherwise }\end{cases}
$$

is good.

Case $3:\left[G: H_{0}\right]=2,\left[G: K_{0}\right]=2$. Also this case splits into subcases.

SUBCASE 3a: The equations are

$$
\begin{array}{ll}
\varepsilon_{1} i_{1}+\ldots+\varepsilon_{r} i_{r} \equiv 0(\bmod 4), & \varepsilon_{h}= \pm 1 \\
\delta_{1} i_{1}+\ldots+\delta_{r} i_{r} \equiv 0(\bmod 4), & \delta_{h}= \pm 1
\end{array}
$$

in this case all primes $p_{h}$ are congruent to $1 \bmod 4$.

The $r$-tuple $i_{1}=2, i_{\lambda}=4$ for $\lambda>1$ is good.

SubCASE $3 b$ : The equations are

$$
\begin{gathered}
\varepsilon_{1} i_{1}+\ldots+\varepsilon_{r} i_{r} \equiv 0(\bmod 4), \quad \varepsilon_{h}= \pm 1 \\
\sum_{h \in S} i_{h} \equiv 0(\bmod 2), \quad S \neq \emptyset,\{1, \ldots, r\}
\end{gathered}
$$

in this case all primes $p_{h}$ are congruent to $1 \bmod 4$.

Let $h \in S, j \notin S$; at least one of the $r$-tuples

$$
i_{h}= \pm 1, i_{j}=1, i_{\lambda}=2 \text { for } \lambda \neq h, j
$$

is good.

SubCASE 3c: The equations are

$$
\begin{aligned}
& \sum_{h \in S} i_{h} \equiv 0(\bmod 2), \quad S \neq \emptyset,\{1, \ldots, r\}, \\
& \sum_{h \in T} i_{h} \equiv 0(\bmod 2), \quad T \neq \emptyset,\{1, \ldots, r\} .
\end{aligned}
$$


We may of course suppose that $S \neq T$ and, possibly interchanging the roles of $S, T$ and their complementary sets, that $1 \notin S \cup T$.

If $S \cap T \neq \emptyset$, let $h$ be an index such that $h \in S \cap T$. The $r$-tuple

$$
i_{1}=i_{h}=1, i_{\lambda}=2 \text { for } \lambda \neq 1, h
$$

is good.

Suppose then that $S \cap T=\emptyset$. If there exists $h>1$ such that $h \notin S \cup T$, let $j_{1} \in S, j_{2} \in T$; the $r$-tuple

$$
i_{1}=i_{h}=i_{j_{1}}=i_{j_{2}}=1, i_{\lambda}=2 \text { for } \lambda \neq 1, h, j_{1}, j_{2}
$$

is good.

If $q_{1} \neq 3$, we can get the same conclusion by looking at the $r$-tuple $i_{j_{1}}=i_{j_{2}}=1, i_{\lambda}=2$ for $\lambda \neq j_{1}, j_{2}$.

So we are left with the case when $q_{1}=3, S \cap T=\emptyset, S \cup T=\{2, \ldots, r\}$, thereby proving Lemma 3.

With the notation of Lemma 3, suppose next that $Q=3 Q_{1} Q_{2}$, and that $H$ is a subgroup of $G$ contained in $H_{0}$. We have the following

Lemma 4. Let $Q$ be odd, $Q=3 Q_{1} Q_{2}$, with $Q_{1}, Q_{2}>1$ and $\left(3, Q_{1}\right)=$ $\left(3, Q_{2}\right)=\left(Q_{1}, Q_{2}\right)=1$. Then the set $X$ of Dirichlet characters of maximal conductor $Q$ is not contained in $H \cup K_{0}$ unless $Q_{1}=5$ and $H$ coincides with the subgroup $H_{1}$ defined by the equation

$$
i_{2}+2 i_{3}+\ldots+2 i_{r} \equiv 0(\bmod 4) .
$$

Proof. By Lemma 3, we have $X \not \subset H \cup K_{0}$ whenever $H$ is contained in a maximal subgroup of $G$ of odd index; hence we may only consider the case when $H$ is contained in a maximal subgroup of $H_{0}$ of index 2 .

The group $H_{0} / 2 H_{0}$ has order $2^{r-1}$ if all primes $p_{h}$ with $h \in S$ are congruent to $1 \bmod 4$ and order $2^{r-2}$ otherwise. In any case there are $2^{r-2}-1$ subgroups of $H_{0}$ of index 2 given by the equations

$$
\sum_{h \in U} i_{h} \equiv 0(\bmod 2)
$$

where $U$ is a subset of $\{1, \ldots, r\}$ different from $\emptyset,\{1, \ldots, r\}, S, T$, and two sets $U$ and $U^{\prime}$ give rise to the same subgroup if and only if $U \cap S, U^{\prime} \cap S$ and $U \cap T, U^{\prime} \cap T$ are either equal or complementary in $S$ and $T$ respectively.

In the case when there exists $h \in S$ with $p_{h} \equiv 3(\bmod 4)$, these are all possible equations of subgroups of $H_{0}$ of index 2. But since necessarily in an equation of this type we must have $U \neq S, T$, the argument of the proof of Lemma 3 shows that we are done in this case.

In the case when $p_{h}$ is congruent to $1 \bmod 4$ for all $h \in S$, renumber the indices $2, \ldots, r$ so that $S=\{2, \ldots, s\}, T=\{s+1, \ldots, r\}$ and $p_{2}<\ldots<p_{s}$, $p_{s+1}<\ldots<p_{r}$. Then the other $2^{r-2}$ subgroups of index 2 are given by the 
equations

$$
\varepsilon_{2} i_{2}+\ldots+\varepsilon_{s} i_{s}+\delta_{s+1} i_{s+1}+\ldots+\delta_{r} i_{r} \equiv 0(\bmod 4),
$$

where $\varepsilon_{h}= \pm 1, \delta_{h}=0,2$, and two such equations give rise to the same subgroup if and only if they are equal or obtained from one another by changing all signs.

If $|S| \geq 2$, hence $s \geq 3$, then at least one of the $r$-tuples given by

$$
i_{1}=i_{r}=1, i_{2}=2, i_{3}=2,4, i_{\lambda}=4 \text { for } \lambda \neq 1,2,3, r
$$

is good.

If $s=2$ and $q_{2} \neq 5$, then at least one of the $r$-tuples

$$
i_{1}=i_{r}=1, i_{2}=2,4, i_{\lambda}=4 \text { for } \lambda \neq 1,2, r
$$

is good.

Suppose now that $s=2$ and $q_{2}=5$. If there exists $h>2$ such that $\delta_{h}=0$, then the $r$-tuple given by

$$
i_{1}=1=i_{h}=1, i_{\lambda}=2 \text { for } \lambda \neq 1, h
$$

is good.

Hence the only possibility is that $Q_{1}=5$ and that $H_{1}$ is the unique maximal subgroup of $H_{0}$ containing $H$.

If $H=H_{1}$, then in fact we have $X \subset H_{1} \cup K_{0}$. If $H$ is properly contained in $H_{1}$, then in fact it must be contained in a subgroup of $H_{1}$ of index 2 . As before, we can check that $H_{1} / 2 H_{1}$ has order $2^{r-2}$ and that all its subgroups of index 2 are given by equations of type

$$
\sum_{h \in V} i_{h} \equiv 0(\bmod 2)
$$

where $V$ is a non-empty subset of $\{3, \ldots, r\}$. Let $h \in V$. Then the $r$-tuple given by

$$
i_{1}=i_{h}=1, i_{\lambda}=2 \text { for } \lambda \neq 1, h
$$

is then good, and this concludes the proof of the lemma.

Consider now the case when all $m, n-m, n$ are coprime to $Q$. Notice that $Q$ must be odd in this case, since at least one of the three numbers $m, n-m, n$ is even.

Let $z$ be the solution $\bmod Q$ of the congruence $m \equiv z n(\bmod Q)$. Let $H, K$ be the subgroups of $G$ defined by the equations $\chi(z)=\chi(1-z)=1$ and $\chi(k)=1$, respectively. We have the following

Lemma 5. If $Q>3$ is odd and $k \neq \pm 1$ then the set $X$ of Dirichlet even characters of maximal conductor $Q$ is not contained in $H \cup K$.

Proof. By Lemma 2, the condition $k \neq \pm 1$ implies that $K \neq G$. Lemma 3 shows that we only have to take care of the case when $Q=3 Q_{1} Q_{2}$ with 
$Q_{1}, Q_{2}>1,\left(3, Q_{1}\right)=\left(3, Q_{2}\right)=\left(Q_{1}, Q_{2}\right)=1$ and the subgroups $H, K$ are contained in maximal subgroups $H_{0}, K_{0}$ defined by the equations

$$
\sum_{h \in S} i_{h} \equiv 0(\bmod 2), \quad \sum_{h \in T} i_{h} \equiv 0(\bmod 2)
$$

respectively, where $S=\left\{h: p_{h} \mid Q_{1}\right\}$ and $T=\left\{h: p_{h} \mid Q_{2}\right\}$.

However, we note that $H$ cannot coincide with the subgroup $H_{0}$ : in fact, the set $\left\{x \in \mathbb{Z} \mid \chi(x)=1 \forall \chi \in H_{0}\right\}$ is contained in the set $x \equiv \pm 1\left(\bmod Q_{1}\right)$ in this case, and it is immediately seen that $z$ and $1-z$ cannot both lie in this set (note that $Q_{1}>3$ ). So it remains to consider the case when $H$ is properly contained in $H_{0}$, and, by Lemma 4 , only the case when $H=H_{1}$, i.e. the subgroup defined by the equation

$$
i_{2}+2 i_{3}+\ldots+2 i_{r} \equiv 0(\bmod 4) .
$$

One easily sees that the set $\left\{x \in \mathbb{Z} \mid \chi(x)=1 \forall \chi \in H_{1}\right\}$ is contained in the set $\left\{x \in \mathbb{Z} \mid x \equiv \pm 1\left(\bmod Q_{2}\right)\right\}$, and again it is not possible that both $z$ and $1-z$ belong to this set. This concludes the proof of the lemma.

Proposition 1. Let $\zeta_{1}=\zeta$ be a primitive $Q$ th root of unity and $\zeta_{2}=\zeta^{k}$. Assume that $(m, Q)=(n-m, Q)=(n, Q)=1$, whence, in particular, that $Q$ is odd. If $(1)$ holds, then $k \equiv \pm 1(\bmod Q)$.

Proof. Consider all relations (7) relative to characters of maximal conductor. They have the form

$$
(1-\chi(k))(m \chi(m)+(n-m) \chi(n-m)-n \chi(n))=0 .
$$

We look when the term inside the second parentheses is zero, i.e. when

$$
m \chi(m)+(n-m) \chi(n-m)-n \chi(n)=0 .
$$

In our notation, this amounts to studying the vanishing of the quantity $m \chi(z)+(n-m) \chi(1-z)-n$. Since $|\chi(z)|=|\chi(1-z)|=1$, this vanishes if and only if $\chi(z)=\chi(1-z)=1$. Since $H$ is a proper subgroup of $G$, Lemma 5 implies that $K=G$ and, by Corollary $1(\mathrm{c}), k \equiv \pm 1(\bmod Q)$.

Proposition 2. Let $\zeta_{1}=\zeta, \zeta_{2}=\zeta^{k}$. Suppose that $Q \not \equiv 2(\bmod 4)$, and that exactly two of the numbers $(m, Q),(n-m, Q),(n, Q)$ are equal to 1 . If (1) holds, then $k \equiv \pm 1(\bmod Q)$.

Proof. By symmetry, we may consider only the case when $(m, Q)=$ $(n-m, Q)=1$ and $(n, Q)>1$. For an even character of maximal conductor $\chi$, relations $(7)$ take the form

$$
(1-\chi(k))(m \chi(m)+(n-m) \chi(n-m))=0 .
$$

Now $m \chi(m)+(n-m) \chi(n-m)=0$ implies $|m|=|n-m|$, hence $n=2 m$ and $2 m \chi(m)=0$, a contradiction. This means that we must have $\chi(k)=1$ for 
all even characters of maximal order. By Corollary 1, the conclusion follows if $Q$ is not of the form $Q=2^{a} \cdot 3, a \geq 3$.

If $Q=2^{a} \cdot 3, a \geq 3$, we need to exclude the case when $k= \pm i$ (with the notation of Corollary 1$)$. Consider a generator $\chi_{0}$ of the even characters $\bmod 2^{a}$; we have $\chi_{0}( \pm i)=-1$ and the relation $Y\left(\chi_{0}, R\right)=0$ is

$$
\frac{1}{\phi\left(2^{a}\right)} \sum_{\substack{x=1 \\\left(x, 2^{a}\right)=1}}^{2^{a}-1} \chi_{0}(x) C_{3 x}+\frac{1}{\phi\left(2^{a} \cdot 3\right)}\left(1-\bar{\chi}_{0}(3)\right) \sum_{\substack{x=1 \\\left(x, 2^{a} \cdot 3\right)=1}}^{2^{a} \cdot 3-1} \chi_{0}(x) C_{x}=0
$$

Since $2 \mid n$ and $3 \nmid m(n-m)$ all the equations $3 x=m, n-m,-n, 3 x=$ $\mp i m, \mp i(n-m), \pm i n$ are unsolvable under the condition $\left(x, 2^{a}\right)=1$; moreover, $\chi_{0}(n)=\chi_{0}( \pm i n)=0$. So equation (14) becomes

$$
2\left(1-\bar{\chi}_{0}(3)\right)(m \chi(m)+(n-m) \chi(n-m))=0,
$$

which is impossible since $\chi_{0}(3) \neq 1$.

Proposition 3. Let $\zeta_{1}=\zeta, \zeta_{2}=\zeta^{k}$. Suppose that $Q \not \equiv 2(\bmod 4)$, and that exactly one of the numbers $(m, Q),(n-m, Q),(n, Q)$ is equal to 1 . If (1) holds, then $k \equiv \pm 1(\bmod Q)$.

Proof. By symmetry, we may only consider the case when $(m, Q)=1$, $(n-m, Q)>1$ and $(n, Q)>1$. For any even character of maximal conductor $Q$, relation $(7)$ reads

$$
(1-\chi(k)) \chi(m)=0 .
$$

By Corollary 1, we need only exclude the case when $Q=2^{a} \cdot 3, a \geq 3$, and $k= \pm i$. We consider again the character $\chi_{0}$. Exactly one number between $n$ and $n-m$ is divisible by 2 , whereas the other one is divisible by 3 . Again we deal only with the case when $2 \mid n$ and $3 \mid n-m$, the other case being similar. Equation (14) becomes

$$
\frac{2}{\phi\left(2^{a}\right)}(n-m) \chi_{0}\left(\frac{n-m}{3}\right)+\frac{2}{\phi\left(2^{a} \cdot 3\right)}\left(1-\bar{\chi}_{0}(3)\right) m \chi_{0}(m)=0
$$

or, equivalently,

$$
2(n-m) \chi_{0}\left(\frac{n-m}{3}\right)+\left(1-\bar{\chi}_{0}(3)\right) m \chi_{0}(m)=0 .
$$

This last equation implies that $\left|1-\bar{\chi}_{0}(3)\right|=2(n-m) / m$; but $1-\chi_{0}(3)$ is an algebraic integer, so if its absolute value is rational then it must be an integer, and in fact it can only be $0,1,2$. All possibilities are easily excluded under our hypotheses, so the proposition follows.

Proposition 4. Let $\zeta_{1}=\zeta, \zeta_{2}=\zeta^{k}$. Suppose that $Q \neq \equiv 2(\bmod 4)$, and that none of the numbers $m, n-m, n$ is coprime to $Q$. If (1) holds, then $k \equiv \pm 1(\bmod Q)$. 
Proof. Let $(m, Q)=p_{1}^{\alpha_{1}} \ldots p_{r}^{\alpha_{r}}, m=(m, Q) m^{*}, Q=(m, Q) f_{m}$, so that $\left(m^{*}, f_{m}\right)=1$, whereas $\left(n-m, f_{m}\right)>1$ and $\left(n, f_{m}\right)>1$. Define $f_{n-m}$ and $f_{n}$ similarly. All numbers $f_{m}, f_{n-m}, f_{n}$ are divisible by at least two prime factors and at least two of them, say $f_{m}$ and $f_{n}$, are divisible by the same power of 2 as $Q$. Consider even characters $\chi$ of conductor $f=f_{m}$.

Let $d$ be an integer such that $f_{m}|d| Q$, and write $d=p_{1}^{i_{1}} \ldots p_{r}^{i_{r}} f$. The equations $(Q / d) x=n, k n$ are not solvable under the given conditions, since $(Q / d, n)=1$ and a possible solution would satisfy $x \equiv 0(\bmod n)$, hence $\left(x, f_{m}\right)>1$ and a fortiori $(x, d)>1$; the same argument shows that the equations $(Q / d) x=n-m, k(n-m)$ are not solvable with $(x, d)=1$. The equation $(Q / d) x=p_{1}^{\alpha_{1}-i_{1}} \ldots p_{r}^{\alpha_{r}-i_{r}} x=m$ has solution $x=p_{1}^{i_{1}} \ldots p_{r}^{i_{r}} m^{*}$, but this solution is coprime to $d$ only if $i_{1}=\ldots=i_{r}=0$, i.e. if $d=f_{m}$. Hence relations (7) for such characters become

$$
\frac{1}{\phi\left(f_{m}\right)}(1-\chi(k)) m \chi\left(m^{*}\right)=0
$$

and we can obtain a similar expression by considering the conductor $f_{n}$. Since at most one of $f_{m}$ and $f_{n}$, say $f_{m}$, can be of the form $2^{a} \cdot 3, a \geq 3$, we get the system

$$
\left\{\begin{array}{l}
k \equiv \pm 1( \pm i)\left(\bmod f_{m}\right) \\
k \equiv \pm 1\left(\bmod f_{n}\right) .
\end{array}\right.
$$

But the solutions of the single congruences must agree modulo $\left(f_{m}, f_{n}\right)>1$ and $\left[f_{m}, f_{n}\right]=Q$, hence we get $k \equiv \pm 1(\bmod Q)$.

4.2. The case $Q \equiv 2(\bmod 4)$. Consider the case when $Q=2 Q^{\prime}, Q^{\prime}$ odd; then exactly one of $m, n-m, n$ is even, say $n=2 n_{1}$. By this assumption we lose some of the symmetries among the numbers $m, n-m,-n$, hence throughout this subsection the number $-n$ will be given a distinguished role. As remarked above, we can suppose that $Q^{\prime}>3$.

Proposition 5. Let $\zeta_{1}=\zeta, \zeta_{2}=\zeta^{k}, k$ odd, and $Q=2 Q^{\prime}, Q^{\prime}$ odd. Suppose moreover that $\left(m, Q^{\prime}\right)=\left(n-m, Q^{\prime}\right)=\left(n, Q^{\prime}\right)=1$. If (1) holds, then $k \equiv \pm 1(\bmod Q)$, except for the case $Q^{\prime}=5,\{4 m, 4(n-m)\}=\{n, 3 n\}$, where $k$ can be any number coprime to 10.

Proof. For even characters $\chi$ of modulus $Q^{\prime}$ relations (7) give

$$
(1-\bar{\chi}(2)) \sum_{\substack{x=1 \\(x, Q)=1}}^{Q} \chi(x) C_{x}+\sum_{\substack{x=1 \\\left(x, Q^{\prime}\right)=1}}^{Q^{\prime}} \chi(x) C_{2 x}=0 .
$$

If $k$ is odd, this becomes

$$
(1-\chi(k))\left[(1-\bar{\chi}(2))(m \chi(m)+(n-m) \chi(n-m))-n \chi\left(n_{1}\right)\right]=0 .
$$


We first examine the characters $\chi$ for which

$$
(1-\bar{\chi}(2))(m \chi(m)+(n-m) \chi(n-m))-n \chi\left(n_{1}\right)=0 .
$$

LEMMA 6. If equation (16) holds, then either

$$
\chi(2)=\chi(3)=-1 \quad \text { and } \quad\{4 m, 4(n-m)\}=\{n, 3 n\}
$$

or

$$
\chi(2)=\zeta_{6} \quad \text { and } \quad \chi\left(n_{1}\right)=\zeta_{6} \chi(m)=\zeta_{6} \chi(n-m) .
$$

Proof. Let $d$ be the order of such a $\chi, \zeta_{d}$ be a primitive $d$ th root of unity and consider the congruence $\bmod 2 \mathbb{Z}\left[\zeta_{d}\right]$; since $m$ is odd, we obtain

$$
(1-\bar{\chi}(2))(\chi(m)-\chi(n-m)) \equiv 0\left(\bmod 2 \mathbb{Z}\left[\zeta_{d}\right]\right) .
$$

Let $(2)=\left(\mathcal{P}_{1} \ldots \mathcal{P}_{s}\right)^{e}$ be the factorization of the ideal (2) into prime ideals (notice that $e$ is a power of 2). We observe that if a number of the form $1-\zeta_{d}^{a}$ belongs to $\mathcal{P}_{h}^{i}$ for some $h, i$, then all its conjugates, being its multiples, belong to $\mathcal{P}_{h}^{i}$; consequently, $1-\zeta_{d}^{a} \in \mathcal{P}_{j}^{i}$ for all $j$, since the prime ideals $\mathcal{P}_{j}$ are all conjugate under the action of the Galois group $\operatorname{Gal}\left(\mathbb{Q}\left(\zeta_{d}\right) / \mathbb{Q}\right)$.

Hence at least one of the numbers $(1-\bar{\chi}(2))^{2}$ and $(\chi(m)-\chi(n-m))^{2}$ belongs to the ideal (2).

CASE 1: $1-(\bar{\chi}(2))^{2} \in(2)$. In this case we have $\chi(2)= \pm 1, \pm i$, where $i^{2}=-1$. If $\chi(2)=1$ equation (16) is clearly impossible. If $\chi(2)=-1$, taking the squares of absolute values in (16) we get

$$
4\left[m^{2}+(n-m)^{2}+m(n-m) \cdot 2 \Re(\chi(m) \bar{\chi}(n-m))\right]=n^{2} .
$$

It follows that the algebraic integer $\theta=2 \Re(\chi(m) \bar{\chi}(n-m))$ is a rational number, hence $\theta \in\{-2,-1,0,1,2\}$. We get

$$
(8-4 \theta) m^{2}+(4 \theta-8) m n+3 n^{2}=0 .
$$

This equation can have integer solutions only if $\theta=-2,-1,2$. If $\theta=2$ then $n=0$, impossible. If $\theta=-1$, then $n=2 m$, but in this case $n-m=m$, hence $\chi(m)=\chi(n-m), \chi(m) \bar{\chi}(m-n)=1$ and $\theta=2$, a contradiction. If $\theta=-2$, then either $4 m=n$ and $4(n-m)=3 n$ or $4 m=3 n$ and $4(n-m)=n$, and therefore $\chi(3)=-1$.

If $\chi(2)= \pm i$, taking the squares of absolute values in (16) we get

$$
2\left(m^{2}+(n-m)^{2}+\theta m(n-m)\right)=n^{2} .
$$

This last equation has rational solutions only if $\theta=0,2$. If $\theta=0$ we get $n=2 m, n-m=m=n_{1}$ and

$$
(1 \pm i) n \chi\left(n_{1}\right)=n \chi\left(n_{1}\right),
$$

a contradiction. If $\theta=2$ we get $n=0$, also a contradiction.

It follows that this case implies $\chi(2)=\chi(3)=-1,\{4 m, 4(n-m)\}=$ $\{n, 3 n\}$. 
CASE 2: $(\chi(m))^{2}-(\chi(n-m))^{2} \in(2)$. This implies that either $\chi(m)=$ $\pm \chi(n-m)$ or $\chi(m)=i \chi(n-m)$, where $i^{2}=-1$. In the latter case the greatest common divisor of the ideals $((1 \pm i) \chi(m))$ and $(2)$ is $(1 \pm i)=$ $\left(\mathcal{P}_{1} \ldots \mathcal{P}_{s}\right)^{e / 2}$, hence we fall again into Case 1.

If $\chi(m)=-\chi(n-m)$, then from (16) we have

$$
(1-\bar{\chi}(2))(2 m-n) \chi(m)=n \chi\left(n_{1}\right) .
$$

This implies again that $|1-\bar{\chi}(2)| \in\{0,1,2\}$. The case when $1-\bar{\chi}(2)=0$, hence $\chi(2)=1$, is excluded trivially. If $|1-\bar{\chi}(2)|=1$, then we get $|2 m-n|$ $=|n|$, which implies either $n=m$ or $m=0$, both cases being impossible. If $|1-\bar{\chi}(2)|=2$, hence $\chi(2)=-1$, we get $|4 m-2 n|=|n|$, and considering both signs we get $\{4 m, 4(n-m)\}=\{n, 3 n\}$, and consequently $\chi(3)=-1$.

If $\chi(m)=\chi(n-m)$, we obtain

$$
(1-\bar{\chi}(2)) n \chi(m)=n \chi\left(n_{1}\right),
$$

whence $\chi(2)=1-\bar{\chi}(2)=\zeta_{6}$ and $\chi\left(n_{1}\right)=\zeta_{6} \chi(m)=\zeta_{6} \chi(n-m)$.

Let $X$ be the set of even Dirichlet characters of conductor $Q^{\prime}$ and let $Z$ be the subset of even characters of conductor $Q^{\prime}$ having either property (17) or (18). Moreover, let $H$ be the subgroup of even Dirichlet characters mod $Q^{\prime}$ generated by $Z$ and let $K$ be the subgroup of even Dirichlet characters $\bmod Q^{\prime}$ defined by the equation $\chi(k)=1$. Equation (16) holds for all even characters of conductor $Q^{\prime}$ only if $X \subset Z \cup K$, whence clearly only if $X \subset$ $H \cup K$.

Suppose that $K \neq G$, and consider first the case when $H$ is the full group of even Dirichlet characters $\bmod Q^{\prime}$. Since both equations (17) and (18) imply that $\chi(2)^{6}=1$, this can only happen if $2^{6} \equiv \pm 1\left(\bmod Q^{\prime}\right)$, i.e. $Q^{\prime} \mid 63$ or $Q^{\prime} \mid 65$.

Lemma 7. If $Q^{\prime} \neq 3,5$ and either $Q^{\prime} \mid 63$ or $Q^{\prime} \mid 65$, then there exists a subset $Y$ of the set $X$ of even primitive characters mod $Q^{\prime}$ such that:

(i) for all $\chi \in Y$ relation (16) does not hold;

(ii) $Y$ generates the full group $G$ of even Dirichlet characters mod $Q^{\prime}$.

Proof. Direct checking or computer search.

By Lemma 7, we immediately conclude that $k \equiv \pm 1\left(\bmod Q^{\prime}\right)$ if $Q^{\prime} \mid 64 \pm 1$ except for the case when $Q^{\prime}=5$ and $\{4 m, 4(n-m)\}=\{n, 3 n\}$.

Let $Q^{\prime}$ be such that $Q^{\prime} \nmid 64 \pm 1$ and suppose that $K \neq G$. By Lemma 3, and with the notation of that lemma, $X \not \subset H \cup K$ unless $Q^{\prime}=3 Q_{1}^{\prime} Q_{2}^{\prime}, H$ is contained in the maximal subgroup $H_{0}$ given by those characters whose restriction $\bmod Q_{1}^{\prime}$ is the full group of even characters $\bmod Q_{1}^{\prime}$, and $K$ is contained in the maximal subgroup $K_{0}$ given by those characters whose restriction $\bmod Q_{2}^{\prime}$ is the full group of even characters $\bmod Q_{2}^{\prime}$.

Lemma 8. If $H=H_{0}$, then $X \not \subset Z \cup K$. 
Proof. By (17) and (18), $H=H_{0}$ implies that $64 \equiv \pm 1\left(\bmod Q_{1}^{\prime}\right)$. Since $\left(3, Q_{2}^{\prime}\right)=1$, we can have $3 Q_{2}^{\prime} \mid 64 \pm 1$ only if $Q_{2}^{\prime}=7$.

CASE 1: $Q_{2}^{\prime} \neq 7$. By Lemma 2 , the set of primitive even characters mod $3 Q_{2}^{\prime}$ generates the group of all even Dirichlet characters mod $3 Q_{2}^{\prime}$, hence there exists an even character $\chi$ of conductor $3 Q_{2}^{\prime}$ such that $\chi(2) \notin \mu_{6}$. Letting $\chi^{\prime}$ be any even character of conductor $Q_{1}^{\prime}$, we get $\chi^{\prime} \chi(2) \notin \mu_{6}$, hence neither (17) nor (18) is satisfied; moreover, the restriction of $\chi^{\prime} \chi \bmod$ $Q_{2}^{\prime}$ is odd, so $\chi^{\prime} \chi \notin K$.

CASE 2: $Q_{2}^{\prime}=7$. By direct checking, we get $Q_{1}^{\prime} \in\{5,13,65\}$. For all these cases, it is an exercise to find characters $\chi$ of conductor $Q^{\prime}$ for which $\chi(2)=\zeta_{3}$, whence $\chi \notin Z \cup K$.

If $H$ is strictly contained in $H_{0}$, then Lemma 4 shows again that $X \not \subset$ $H \cup K$ unless $Q_{1}^{\prime}=5, Q^{\prime}=3 \cdot 5 \cdot Q_{2}^{\prime},\left(15, Q_{2}^{\prime}\right)=1, H$ coincides with the subgroup of the characters defined by equation (12) and $K$ is contained in the subgroup of those characters whose restriction mod 15 is even. We claim that also in this case $X \not \subset Z \cup K$.

Definition 1. Here and in what follows, if $q>2$ is a prime power we shall denote by $\chi_{q}$ an (odd) generator of the group of characters $\bmod q$.

Consider characters of type $\chi_{3} \chi_{5}^{2} \chi$, where $\chi$ is an odd primitive character $\bmod Q_{2}^{\prime}$. Since $\chi_{3} \chi_{5}^{2}(2)=1$, it is enough to show that there exists an odd primitive character $\chi \bmod Q_{2}^{\prime}$ such that $\chi(2) \neq-1, \zeta_{6}$. Clearly the odd primitive characters mod $Q_{2}^{\prime}$ generate the full group $\widehat{G}$ of Dirichlet characters $\bmod Q_{2}^{\prime}$ (remember that $Q_{2}^{\prime}$ is odd), hence the existence of such a $\chi$ is guaranteed unless $Q_{2}^{\prime} \nmid 63=2^{6}-1$, which, in our situation, leaves only the case $Q_{2}^{\prime}=7$. In this case we can take $\chi=\chi_{3} \chi_{5}^{2} \chi_{7}$, and the claim is proved.

It follows that, if $H \neq G$, then $X \subset H \cup K$ implies $K=G$, whence $k \equiv \pm 1\left(\bmod Q^{\prime}\right)$. Together with Lemma 7 , this concludes the proof of Proposition 5.

REMARK 2. We make it explicit what happens in the case $Q^{\prime}=5$ and $\{4 m, 4(n-m)\}=\{n, 3 n\}$. The assumptions in Proposition 5 say that $k$ is odd; moreover, in this case $k \not \equiv 5(\bmod 10)$, since otherwise we would have $\zeta^{k n}-1=0$. The condition $\chi(2)=\chi(3)=-1$ is clearly satisfied for the only non-trivial even character $\chi \bmod 5$. The condition $\{4 m, 4(n-m)\}=\{n, 3 n\}$ implies that $n=4 x$ for some integer $x$; moreover, $x$ cannot be divisible by 5 (otherwise $n$ would be divisible by 10 and $\zeta^{k n}-1=0$ ) and $x$ must be odd (otherwise $m, n-m, n$ would be all even). It follows that $\{m, n-m\}=$ $\{x, 3 x\}$ and $n=4 x$ for some $x$ coprime to 10 . In all these cases, it is easy to verify that the two terms in (1) coincide, their common value being $1 / 5^{x}$. Finally, by the symmetry among the numbers $m, n-m,-n$ remarked in the 
introduction, equation (1) holds whenever $\{m, n-m,-n\}=\{x, 3 x,-4 x\}$ and $(x, 10)=(k, 10)=1$.

Proposition 6. Let $\zeta_{1}=\zeta, \zeta_{2}=\zeta^{k}, k$ even, and $Q=2 Q^{\prime}, Q^{\prime}$ odd. Suppose that $\left(m, Q^{\prime}\right)=\left(n-m, Q^{\prime}\right)=\left(n_{1}, Q^{\prime}\right)=1$. Then equation $(1)$ does not hold.

Proof. Let $k=2 k_{1}$. For even characters $\chi$ of modulus $Q^{\prime}$ Ennola's relations (7) give

$$
\left[1-\bar{\chi}(2)-\chi\left(k_{1}\right)\right](m \chi(m)+(n-m) \chi(n-m))=(1-\chi(k)) n \chi\left(n_{1}\right) .
$$

Suppose first that $\chi\left(k_{1}\right)=0$, whence $\chi(k)=0$. Then equation (20) reduces to equation (16). Proposition 5 shows that this equation can be satisfied for all characters only if $Q^{\prime}=5$. But in this case $\chi(k)$ cannot be zero, since otherwise $k$ would be divisible by 10 . Hence in the rest of the proof we shall assume $\chi\left(k_{1}\right) \neq 0$.

LEMMA 9. If equation (20) holds, then $\chi(2)$ belongs either to $\mu_{6}$ or to $\mu_{10}$. Moreover, if $\chi(2)= \pm 1$, then $\chi$ has order multiple of 6 .

Proof. Consider, as in Lemma 6, the congruence modulo the ideal (2) in $\mathbb{Z}\left[\zeta_{d}\right]$ :

$$
\left(1-\bar{\chi}(2)-\chi\left(k_{1}\right)\right)(\chi(m)-\chi(n-m)) \equiv 0(\bmod (2)) .
$$

The greatest common divisor between the ideals $(\chi(m)-\chi(n-m))$ and $(2)$ is of the form $\left(\mathcal{P}_{1} \ldots \mathcal{P}_{s}\right)^{e^{\prime}}$, hence we see again that either $\left(1-\bar{\chi}(2)-\chi\left(k_{1}\right)\right)^{2}$ or $(\chi(m)-\chi(n-m))^{2}$ must be divisible by 2 .

CASE 1: $1-(\bar{\chi}(2))^{2}-\left(\chi\left(k_{1}\right)\right)^{2} \in(2)$. If we have $1+\zeta_{d}^{a}+\zeta_{d}^{b} \in(2)$, then the same is true for its complex conjugate, hence $1+\zeta_{d}^{b-a}+\zeta_{d}^{b} \in(2)$ and $\zeta_{d}^{a}+\zeta_{d}^{b-a} \in(2)$. This means that $\zeta_{d}^{a}= \pm \zeta_{d}^{b-a}, \zeta_{d}^{b}=\zeta_{d}^{a} \zeta_{d}^{b-a}= \pm \zeta^{2 a}$ and $\zeta^{3 a}= \pm 1$. Notice that however $\zeta^{a}$ cannot be itself equal to \pm 1 , hence $\zeta^{a}=\zeta_{3}$ or $\zeta^{a}=\zeta_{6}$.

It follows that $\chi(2)$ must be one of $\zeta_{3}, \zeta_{6}, \zeta_{12}$.

CASE 2: $(\chi(m))^{2}-(\chi(n-m))^{2} \in(2)$. As in Lemma 6, we can suppose that $\chi(m)= \pm \chi(n-m)$.

Suppose that $\chi(m)=\chi(n-m)$. Putting this into (20), we get

$$
\left[1-\bar{\chi}(2)-\chi\left(k_{1}\right)\right] \chi(m)=(1-\chi(k)) \chi\left(n_{1}\right),
$$

giving a 5 -term relation among roots of unity. Suppose first that such a relation is indecomposable. By a theorem of Conway and Jones [2], all the roots of unity involved (i.e. the ratios between two roots of unity occurring in the relation) must belong either to $\mu_{6}$ or to $\mu_{10}$. If the relation is decomposable, then necessarily it must split into two subrelations of lengths 2 and 3 . We leave it as an exercise to the reader to check that all possible splittings give either a contradiction or the conclusion $\chi(2) \in \mu_{6}$. 
Moreover, notice that substituting $\chi(2)=1$ into $(22)$ gives $\chi(k)=$ $\chi\left(k_{1}\right)=\zeta_{6}$ and $\chi(m)=\zeta_{6} \chi\left(n_{1}\right)$; and substituting $\chi(2)=-1$ gives $\chi\left(k_{1}\right)=$ $-\chi(k)=\zeta_{6}$ and again $\chi(m)=\zeta_{6} \chi\left(n_{1}\right)$, whence $\chi$ has order multiple of 6 .

Suppose now that $\chi(m)=-\chi(n-m)$, and substitute this into (20). We get

$$
\left[1-\bar{\chi}(2)-\chi\left(k_{1}\right)\right](2 m-n) \chi(m)=(1-\chi(k)) n \chi\left(n_{1}\right),
$$

yielding a 5-term relation among roots of unity. If the relation is indecomposable, then all roots of unity involved belong either to $\mu_{6}$ or to $\mu_{10}$. If any of the roots of unity involved has order multiple of 5 , then all coefficients must have the same absolute value, hence $|2 m-n|=|n|$, impossible under our hypotheses. Hence necessarily all roots involved, and in particular $\chi(2)$, must belong to $\mu_{6}$. Consider now the case when the relation is decomposable, whence necessarily it splits in one 2-term relation and one 3 -term relation. Both relations must have coefficients of equal absolute value, whence the only possibility is that the two terms are $1, \chi(k)$ and the three terms are $1, \bar{\chi}(2), \chi\left(k_{1}\right)$. This again implies that $\chi(2) \in \mu_{6}$.

Moreover, note that if $\chi(2)=1$ then $\chi(k)=\chi\left(k_{1}\right)$ and $1-\chi(k)$ must be an integer. If $1-\chi(k)=0$ then $2 m-n=0$ and $\chi(m)=\chi(n-m)$, a contradiction. If $1-\chi(k)=1$ then $|2 m-n|=|n|$, impossible under our hypotheses. If $1-\chi(k)=2$, hence $\chi(k)=-1$, we get $(2 m-n) \chi(m)=$ $2 n \chi\left(n_{1}\right)$; it follows that $|2 m-n|=|2 n|$, so either $2 m=3 n$ and $2(n-m)$ $=-n$ or $2 m=-n$ and $2(n-m)=3 n$. In both cases we must have $\chi(3)=-1$ and, substituting into (23), we get a contradiction.

If $\chi(2)=-1$, hence $\chi(k)=-\chi\left(k_{1}\right)$, then taking absolute values we obtain

$$
\left[5-2\left(\chi\left(k_{1}\right)+\bar{\chi}\left(k_{1}\right)\right)\right](2 m-n)^{2}=\left[2+\left(\chi\left(k_{1}\right)+\bar{\chi}\left(k_{1}\right)\right)\right] n^{2} .
$$

It follows that $\chi\left(k_{1}\right)+\bar{\chi}\left(k_{1}\right)$ must lie in the set $\{-2,-1,0,1,2\}$; a direct check shows that the cases when $\chi\left(k_{1}\right)+\bar{\chi}\left(k_{1}\right)=0,-1$ do not have integer solutions $(m, n)$; the case $\chi\left(k_{1}\right)+\bar{\chi}\left(k_{1}\right)=-2$ gives $n=2 m$ and contradicts our hypotheses, the case $\chi\left(k_{1}\right)+\bar{\chi}\left(k_{1}\right)=1$ gives $|2 m-n|=|n|$, contradicting our hypotheses; finally, $\chi\left(k_{1}\right)+\bar{\chi}\left(k_{1}\right)=2$ leads to the equation $(2 m-n) \chi(m)=$ $2 n \chi\left(n_{1}\right)$, already considered above and shown to be contradictory.

To conclude the proof of the lemma, it remains to exclude the case when $\chi(2) \in \mu_{12}$, which occurs in Case 1 .

If $\chi(2)=\zeta_{12}$ and $1-(\bar{\chi}(2))^{2}-\left(\chi\left(k_{1}\right)\right)^{2} \in(2)$, then $\chi\left(k_{1}\right) \in\left\{\zeta_{12}, \zeta_{12}^{4}, \zeta_{12}^{7}\right.$, $\left.\zeta_{12}^{10}\right\}$. The factorization of the ideal $(2)$ in $\mathbb{Z}\left[\zeta_{12}\right]$ is $(2)=\mathcal{P}^{2}=\left(\zeta_{12}^{2}+\zeta_{12}+1\right)^{2}$ and it is easily checked that in all the above cases we have $\left(1-\bar{\chi}(2)-\chi\left(k_{1}\right)\right)$ $=\mathcal{P}$. This means that Case 2 must hold as well. Since $\chi(m)= \pm \chi(n-m)$ imply that $\chi(2)$ belongs either to $\mu_{6}$ or to $\mu_{10}$, it remains to consider the 
case when $\chi(m)= \pm i \chi(n-m)$, where $i^{2}=-1$. For $j \in\{1,4,7,10\}$, let

$$
\begin{aligned}
& A_{j}=\left|1-\bar{\chi}(2)-\chi\left(k_{1}\right)\right|^{2}=\left|1-\zeta_{12}^{-1}-\zeta_{12}^{j}\right|^{2}, \\
& B_{j}=|1-\chi(k)|^{2}=\left|1-\zeta_{12}^{j+1}\right|^{2} .
\end{aligned}
$$

Taking the squares of absolute values in (20) we obtain

$$
A_{j}\left[m^{2}+(n-m)^{2}\right]=B_{j} n^{2} \quad \text { for } j \in\{1,4,7,10\} .
$$

We leave it to the reader to check that the last equation has no integer solutions $(m, n)$ for $j \in\{1,4,7,10\}$.

By Lemma 9, if $Q^{\prime} \nmid 2^{10} \pm 1$ and $Q^{\prime} \nmid 2^{6} \pm 1$ there exist even characters of maximal conductor for which (20) is not true, hence Proposition 6 follows for these values of $Q^{\prime}$. The remaining cases can be settled by a computer search.

Proposition 7. Let $\zeta_{1}=\zeta, \zeta_{2}=\zeta^{k}, Q=2 Q^{\prime}, Q^{\prime}$ odd. Suppose that exactly two of the numbers $m, n-m, n_{1}$ are coprime to $Q$. If equation (1) holds, then $k \equiv \pm 1(\bmod Q)$.

Proof. The relevant equations depend on the parity of $k$ and on which terms are coprime to $Q$. Consider first the case when $k$ is odd. Then we must consider specializations of (15).

CASE 1: The relevant equation is

$$
(1-\chi(k))[(1-\bar{\chi}(2))(m \chi(m)+(n-m) \chi(n-m))]=0 .
$$

We examine when the term inside the square brackets is zero, i.e. when

$$
(1-\bar{\chi}(2))(m \chi(m)+(n-m) \chi(n-m))=0 .
$$

Equation (24) implies that $\chi(2)=1$. The subgroup $H$ of Dirichlet characters with this property is always proper. If $K \neq G$, we can have $X \subset H \cup K$ only if $Q^{\prime}=3 Q_{1}^{\prime} Q_{2}^{\prime}$ and $H$ is contained in the subgroup of those characters whose restriction mod $Q_{1}^{\prime}$ is even. It cannot coincide with this subgroup, since $Q_{1}^{\prime} \neq 3$. If it is properly contained in this subgroup, then the only possibility is that $Q=3 \cdot 5 \cdot Q_{2}^{\prime}$ and $H$ coincides with the subgroup defined by equation (12), while $K$ is contained in the subgroup of those characters whose restriction mod 15 is even.

If this is the case, consider characters of the type $\chi_{3} \chi_{5}^{2} \chi$, where $\chi$ is an odd primitive character mod $Q_{2}^{\prime}$. Since $\chi_{3} \chi_{5}^{2}(2)=1$, it is enough to show that there exists a primitive character $\chi \bmod Q_{2}^{\prime}$ such that $\chi(2) \neq 1$, and this is trivial.

CASE 2: The equation is

$$
(1-\chi(k))\left[(1-\bar{\chi}(2)) m \chi(m)-n \chi\left(n_{1}\right)\right]=0 .
$$


We look again when the term inside the square brackets is zero, i.e. when

$$
(1-\bar{\chi}(2)) m \chi(m)-n \chi\left(n_{1}\right)=0 .
$$

Equation (25) can hold only if the congruence mod (2) is satisfied, i.e. if $\chi(2)= \pm 1$. The subgroup of characters with this property is not the full group $G$ unless $Q^{\prime}=5$. But $Q^{\prime}$ cannot be a prime number, otherwise we would have $Q^{\prime} \mid n-m$, contrary to our assumptions. Hence the subgroup is proper, and we can use the argument above to show that we need only consider the case $Q=3 Q_{1}^{\prime} Q_{2}^{\prime}$ and $H$ is contained in the subgroup of those characters whose restriction $\bmod Q^{\prime}$ is even. Both when $H$ coincides with this subgroup and when it is properly contained in it, we must have $Q_{1}^{\prime}=5$. Remembering that $\chi_{3} \chi_{5}^{2}(2)=1$, this time it is enough to show that there exists an odd primitive character $\chi \bmod Q_{2}^{\prime}$ such that $\chi(2) \neq \pm 1$, and this is again trivial.

Let now $k=2 k_{1}$ be even. Then we must look at specializations of (20).

CASE 3: The equation is

$$
\left(1-\bar{\chi}(2)-\chi\left(k_{1}\right)\right)(m \chi(m)+(n-m) \chi(n-m))=0 .
$$

If $\chi\left(k_{1}\right)=0$, we reduce to equation (24) and the argument of Case 1 applies.

If $\chi\left(k_{1}\right) \neq 0$, then the only possibility is that $\chi(2)=\chi\left(k_{1}\right)=\zeta_{6}$. This leads again to the study of the cases when either $Q^{\prime} \mid 63$ or $Q^{\prime} \mid 65$, which can be dealt with directly.

CASE 4: The equation is

$$
\left(1-\bar{\chi}(2)-\chi\left(k_{1}\right)\right) m \chi(m)=(1-\chi(k)) n \chi\left(n_{1}\right) .
$$

If $\chi\left(k_{1}\right)=0$, then we reduce to (25), and the argument of Case 2 applies.

If $\chi\left(k_{1}\right) \neq 0$, then the congruence $\bmod (2)$ implies that $\chi(2)=\zeta_{3}, \zeta_{6}$, hence $Q^{\prime} \mid 63$ or $Q^{\prime} \mid 65$. A direct check gives the conclusion.

Proposition 8. Let $\zeta_{1}=\zeta, \zeta_{2}=\zeta^{k}, Q=2 Q^{\prime}, Q^{\prime}$ odd. Suppose that exactly one of the numbers $m, n-m, n_{1}$ is coprime to $Q$. If equation (1) holds, then $k \equiv \pm 1(\bmod Q)$.

Proof. Consider first the case when $k$ is odd, and hence specializations of (15).

Case 1: The equation is

$$
(1-\chi(k))[(1-\bar{\chi}(2)) m \chi(m)]=0 .
$$

Use the same argument as in Proposition 7, Case 1.

CASE 2: The equation is

$$
(1-\chi(k)) n \chi\left(n_{1}\right)=0,
$$

which gives immediately the conclusion.

Suppose now that $k=2 k_{1}$ is even, and specialize (20) accordingly. 
CASE 3: The equation is

$$
\left(1-\bar{\chi}(2)-\chi\left(k_{1}\right)\right) m \chi(m)=0
$$

Use the same argument as in Proposition 7, Case 3.

CASE 4: The equation is

$$
(1-\chi(k)) n \chi\left(n_{1}\right)=0 .
$$

This equation can be true for all primitive characters only if $k \equiv \pm 1$ $\left(\bmod Q^{\prime}\right)$. If this is the case, let $\left(m, Q^{\prime}\right)=m_{0}, m=m_{0} m^{*}, Q^{\prime}=m_{0} f_{m}$, so that $\left(m^{*}, f_{m}\right)=1$. Define $(n-m)^{*}$ and $f_{n-m}$ similarly. Since $\left[f_{m}, f_{n-m}\right]=Q^{\prime}$ and $Q^{\prime}$ has at least two distinct prime factors, at least one between $f_{m}$ and $f_{n-m}$, say $f_{m}$, is greater than 3 , hence we may consider non-trivial even characters $\chi$ of conductor $f=f_{m}$. Let $d$ be such that $f|d| Q$. The equation $(Q / d) x=m$ has a solution with $(x, d)=1$ only in the case $d=2 f$ and the solution is $x=m^{*}$; the equation $(Q / d) x=k m$ has a solution with $(x, d)=1$ only if $d=f$ and the solution is $x=k_{1} m^{*}$; the equations $(Q / d) x=n-m, k(n-m)$ do not have solutions with $(x, d)=1$ since $(Q, n-m)=1$ and $(n-m, f)>1$; the equations $(Q / d) x=n, k n$ have solutions with $(x, d)=1$ only if $d=m_{0} f=Q^{\prime}$ and the solutions are $x=n_{1}, k n_{1}$. The corresponding relations (7) give

$$
\frac{1}{\phi(f)}\left(1-\bar{\chi}(2)-\chi\left(k_{1}\right)\right) m \chi\left(m^{*}\right)=\frac{1}{\phi\left(Q^{\prime}\right)}\left(\prod_{p \mid m_{0}}(1-\bar{\chi}(p))\right)(1-\chi(k)) \chi\left(n_{1}\right) \text {. }
$$

Now notice that $k \equiv \pm 1\left(\bmod Q^{\prime}\right)$ implies $k \equiv \pm 1(\bmod f)$, hence $\chi(k)=1$ and the term on the right is zero. Also, since $k=2 k_{1}, \chi\left(k_{1}\right)=\bar{\chi}(2)$, hence we obtain $2 \bar{\chi}(2)=1$, a contradiction.

Proposition 9. Let $\zeta_{1}=\zeta, \zeta_{2}=\zeta^{k}, Q=2 Q^{\prime}, Q^{\prime}$ odd. Suppose that none of the numbers $m, n-m, n_{1}$ is coprime to $Q$. Then $k \equiv \pm 1(\bmod Q)$.

Proof. Let $\left(m, Q^{\prime}\right)=p_{1}^{\alpha_{1}} \ldots p_{r}^{\alpha_{r}}, m=\left(m, Q^{\prime}\right) m^{*}, Q^{\prime}=\left(m, Q^{\prime}\right) f_{m}$, so that $\left(m^{*}, f_{m}\right)=1$, whereas $\left(n-m, f_{m}\right)>1$ and $\left(n, f_{m}\right)>1$. Define $f_{n-m}$ and $f_{n}$ similarly. All numbers $f_{m}, f_{n-m}, f_{n}$ are divisible by at least two prime factors. Consider even characters $\chi$ of conductor $f=f_{m}$.

Let $d$ be an integer such that $f_{m}|d| Q$, and write $d=p_{1}^{i_{1}} \ldots p_{r}^{i_{r}} f$. The equations $(Q / d) x=n, k n$ are not solvable under the given conditions, since $(Q / d, n)=2$ and a possible solution would satisfy $x \equiv 0\left(\bmod n_{1}\right)$, hence $\left(x, f_{m}\right)>1$ and a fortiori $(x, d)>1$; the same argument shows that the equations $(Q / d) x=n-m, k(n-m)$ are not solvable with $(x, d)=1$. The equation $(Q / d) x=\left(2 Q^{\prime} / d\right) x=p_{1}^{\alpha_{1}-i_{1}} \ldots p_{r}^{\alpha_{r}-i_{r}} x=m$ has a solution only if $2 \mid d$ and the solution is $x=p_{1}^{i_{1}} \ldots p_{r}^{i_{r}} m^{*}$; this solution is coprime to $d$ only if 
$i_{1}=\ldots=i_{r}=0$, i.e. if $d=2 f_{m}$. As for the equation $(Q / d) x=\left(2 Q^{\prime} / d\right) x=$ $\mathrm{km}$, there is only one solution of the type required, namely $x=k m^{*}$ for $d=2 f_{m}$ if $k$ is odd, and $x=k_{1} m^{*}$ for $d=f_{m}$ if $k=2 k_{1}$ is even.

Hence relations (7) for such characters become

$$
\begin{cases}(1-\chi(k))(1-\bar{\chi}(2)) m \chi\left(m^{*}\right)=0 & \text { if } k \text { is odd } \\ \left(1-\bar{\chi}(2)-\chi\left(k_{1}\right)\right) m \chi\left(m^{*}\right)=0 & \text { if } k \text { is even. }\end{cases}
$$

By Proposition 8 , we obtain $k \equiv \pm 1\left(\bmod 2 f_{m}\right)$ and, similarly, $k \equiv \pm 1$ $\left(\bmod 2 f_{n-m}\right)$. But the solutions of the single congruences must agree modulo $\left(2 f_{m}, 2 f_{n-m}\right)>2$ and $\left[2 f_{m}, 2 f_{n-m}\right]=Q$, hence we get $k \equiv \pm 1(\bmod Q)$.

REMARK 3. If $Q \not \equiv 2(\bmod 4)$ and at least one of the numbers $m, n-m, n$ is coprime to $Q$, Propositions 1, 2 and 3 show that equation (13) cannot be true for all primitive characters $\bmod Q$. If $Q \equiv 2(\bmod 4)$ and at least one of the numbers $m, n-m, n_{1}$ is coprime to $Q^{\prime}$, Propositions 5, 7 and 8 show that, apart from the exceptions given, equation (16) cannot be true for all primitive characters $\bmod Q^{\prime}$.

We shall use this remark in the proof of Proposition 10.

\section{The case when none of the roots is primitive}

PROPOSITION 10. If neither $\zeta_{1}$ nor $\zeta_{2}$ is a primitive $Q$ th root of unity, then equation (1) does not hold.

Proof. Let $\zeta_{1}=\zeta^{l}$ and $\zeta_{2}=\zeta^{k},(l, Q)=D_{l}>1,(k, Q)=D_{k}>1$, $\left(D_{l}, D_{k}\right)=1$.

Let also $l=D_{l} l^{*}, Q=D_{l} f_{l}$ so that $\left(l^{*}, f_{l}\right)=1$ and $\left(k, f_{l}\right)>1$. Define $f_{k}$ similarly. Note that, since $(l, k, Q)=1$, we have $\left[f_{l}, f_{k}\right]=Q$.

By using relations of type (8), one can easily exclude the finite number of cases when both $f_{l}$ and $f_{k}$ are contained in the set $\{2,3,4,6\}$. By symmetry, we can suppose that $f_{l} \notin\{2,3,4,6\}$. If both $f_{l}$ and $f_{k}$ do not belong to $\{2,3,4,6\}$, one can of course suppose also that $f_{l} \neq 10$. If $f_{k}$ does belong to the set $\{2,3,4,6\}$, one further application of relations (8) leads us to exclude that $f_{l}=10$ and $\{4 m, 4(n-m)\}=\{n, 3 n\}$.

Summarizing, we can assume, by symmetry, that $f_{l}$ satisfies the following condition:

$$
f_{l} \notin\{2,3,4,6\} \text { and, if } f_{l}=10 \text {, then }\{4 m, 4(n-m)\} \neq\{n, 3 n\} .
$$

The following lemma shows that we can also assume that $D_{k} \neq 2,4$.

Lemma 10. If $D_{k}=2,4$ and $f_{l}$ satisfies condition (27), then $D_{l} \neq 2,4$ and we can assume that also $f_{k}$ satisfies condition (27). 
Proof. Since $(k, l, Q)=1, D_{k}=2,4$ implies that $D_{l}$ is odd. Now, as $Q=D_{k} f_{k}$, the lemma is reduced to the verification of a finite number of cases.

Suppose now that $f_{l} \not \equiv 2(\bmod 4)$ and at least one of the numbers $m$, $n-m, n$ is coprime to $f_{l}$. Consider even characters $\chi$ of conductor $f=f_{l}$ and let $d$ be such that $f|d| Q$. The equations $(Q / d) x=k m, k(n-m), k n$ are not solvable under the given conditions, since $(Q / d, k)=1$ and $\left(k, f_{l}\right)>1$. Similarly, the equations $(Q / d) x=l m, l(n-m), n$ can have a solution of the type required only if $d=f_{l}$ (the existence of a solution depending, respectively, on whether or not $\left.\left(m, f_{l}\right)=1,\left(n-m, f_{l}\right)=1,\left(n, f_{l}\right)=1\right)$. In any case we are left with the relations

$$
\chi\left(l^{*}\right)(m \chi(m)+(n-m) \chi(n-m)-n \chi(n))=0 .
$$

Since $\chi\left(l^{*}\right) \neq 0$, these relations cannot hold for all even characters $\chi$ of conductor $f_{l}$ by Remark 3 .

Next, suppose that $f_{l}=2 f_{l}^{\prime} \equiv 2(\bmod 4), n=2 n_{1}$ and that at least one of the numbers $m, n-m, n_{1}$ is coprime to $f_{l}$. Consider even characters $\chi$ of conductor $f=f_{l}^{\prime}$ and let $d$ be such that $f|d| Q$ as before.

The equations $(Q / d) x=k m, k(n-m), k n$ are not solvable under the given conditions, since $(Q / d, k) \leq 2$ and $\left(k, f_{l}\right)>2$. The equations $(Q / d) x=$ $l m, l(n-m)$ can have a solution of the type required only if $d=f_{l}^{\prime}$, and in this case we have $x=l^{*} m, l^{*}(n-m)$. The equation $(Q / d) x=l n$ can have a solution of the type required only if $d=f_{l}$, the solution being $x=l^{*} n_{1}$. Hence relations (7) for these characters give

$$
\chi\left(l^{*}\right)\left[(1-\bar{\chi}(2))(m \chi(m)+(n-m) \chi(n-m))-n \chi\left(n_{1}\right)\right]=0 .
$$

But $\chi\left(l^{*}\right) \neq 0$, and, taking into account that we have supposed $\{4 m$, $4(n-m)\} \neq\{n, 3 n\}$ if $f_{l}=10$, these relations cannot hold for all even characters $\chi$ of conductor $f_{l}^{\prime}$ by Remark 3 .

Assume now that none of the numbers $m, n-m, n$ is coprime to $f_{l}$, and let $D_{m}=\left(m, f_{l}\right)>1, D_{n-m}=\left(n-m, f_{l}\right)>1, D_{n}=\left(n, f_{l}\right)>1$. Clearly we have $\left(D_{m}, D_{n-m}\right)=\left(D_{m}, D_{n}\right)=\left(D_{n-m}, D_{n}\right)=1$. Notice that in this case $f_{l}$ must be divisible by at least three distinct prime factors, and in particular there exists a prime $p_{0} \geq 5$ such that $p_{0} \mid f_{l}$. If $D_{k} \mid 2 D_{m}$, then $D_{k} \nmid 2 D_{n-m}$ and $D_{k} \nmid 2 D_{n}$, otherwise $m$ and $n$ would have a common factor which is a divisor of $Q$ (remember that we have supposed $D_{k} \neq 2,4$ ).

REMARK 4. In what follows we allow complete symmetry among the numbers $m, n-m,-n$, and in particular we do not suppose that $-n$ is the even number among the three.

Possibly interchanging the roles of $m, n-m, n$, we can then suppose that $D_{k} \nmid 2 D_{m}$. Also, since at least two of $m, n-m, n$ have this property, the 
choice can be made so that $p_{0} \nmid D_{m}$. Let $f_{l}=D_{m} f^{*}, m=D_{m} m^{*}$, so that $\left(m^{*}, f^{*}\right)=1$. Now $f^{*}$ is divisible by at least two distinct prime factors, one of which is $p_{0}$. Finally, if any of the numbers $D_{m}, D_{n-m}, D_{n}$ is equal to 2 , we agree to choose $m$ so that $D_{m}=2$, while $D_{n-m}$ and $D_{n}$ are odd.

If $f^{*} \not \equiv 2(\bmod 4)$, consider even primitive characters of conductor $f^{*}$. Letting again $d$ be such that $f^{*}|d| Q$, the equations $(Q / d) x=\mathrm{km}$, $k(n-m), k n$ have no solutions under the given conditions, since $\left(D_{l}, D_{k}\right)=1$ and $D_{k} \nmid D_{m}$ ensure that a possible solution would be divisible by a prime dividing $D_{k}$, and hence would not be coprime to $f^{*}$. The equation $(Q / d) x=$ $l m$ has a solution of the type required only if $d=f^{*}$, the solution being $x=l^{*} m^{*}$, a number coprime to $f^{*}$. The equations $(Q / d) x=l(n-m), l n$ do not have solutions of the type required; in fact, in order that a solution be coprime to $d f^{*}$ we must necessarily have $(d, l)=1$ and, assuming this, the condition $\left(D_{m}, D_{n-m}\right)=\left(D_{m}, D_{n}\right)=1$ implies that a possible solution would have a factor dividing $D_{n-m}, D_{n}$, respectively, and hence would not be coprime to $d f^{*}$. It follows that relations (7) simply tell you that

$$
m \chi\left(l^{*} m^{*}\right)=0
$$

a contradiction.

If $f^{*}=2 f^{\prime *} \equiv 2(\bmod 4)$, consider even primitive characters of conductor $f^{\prime *}$. Letting again $d$ be such that $f^{*}|d| Q$, the equations $(Q / d) x=k m$, $k(n-m), k n$ have no solutions under the given conditions, since $\left(D_{l}, D_{k}\right)=1$ and $D_{k} \nmid 2 D_{m}$ ensure that a possible solution would be divisible by a prime dividing $D_{k}$, and hence would not be coprime to $f^{*}$. The equation $(Q / d) x=$ $l m$ has a solution of the type required only if either $d=f^{*}$ or $d=f^{\prime *}$, depending on whether $m^{*}$ is odd or $m^{*}=2 m_{1}^{*}$ is even; the solution in this case is either $x=l^{*} m^{*}$ or $x=l^{*} m_{1}^{*}$, in any case a number coprime to $f^{\prime *}$. The equations $(Q / d) x=l(n-m), l n$ do not have solutions of the type required; in fact, in order that a solution be coprime to $d f^{*}$ we must necessarily have $(d, l)=1$; assuming this, the conditions $\left(D_{m}, D_{n-m}\right)=\left(D_{m}, D_{n}\right)=1$ and $D_{n-m} \neq 2, D_{n} \neq 2$ imply that $D_{n-m} \nmid 2 D_{m}, D_{n} \nmid 2 D_{m}$, hence a possible solution would have a factor dividing $D_{n-m}, D_{n}$, respectively, and therefore would not be coprime to $d f^{*}$. It follows that relations (7) simply tell you that

$$
\begin{cases}(1-\bar{\chi}(2)) m \chi\left(l^{*} m^{*}\right)=0 & \text { if } m^{*} \text { is odd, } \\ m \chi\left(l^{*} m_{1}^{*}\right)=0 & \text { if } m^{*} \text { is even }\end{cases}
$$

and this is impossible for all even characters $\bmod f^{* *}$ since $f^{\prime *}$ is divisible by $p_{0} \geq 5$.

Acknowledgements. I wish to thank Professor Andrzej Schinzel for suggesting the problem to me and for several helpful comments. Also, I acknowledge the hospitality of the Instytut Matematyczny Polskiej Akademii Nauk, where most of this research was done. 


\section{References}

[1] M. Conrad, On explicit relations between cyclotomic numbers, Acta Arith. 93 (2000), $67-76$.

[2] J. H. Conway and A. J. Jones, Trigonometric diophantine equations (On vanishing sums of roots of unity), ibid. 30 (1976), 229-240.

[3] V. Ennola, On relations between cyclotomic units, J. Number Theory 4 (1972), 236247.

[4] A. Schinzel, On the greatest common divisor of two univariate polynomials, II, this issue, $95-106$.

[5] A. Schinzel, J. Urbanowicz and P. Van Wamelen, Class numbers and short sums of Kronecker symbols, J. Number Theory 78 (1999), 62-84.

[6] C.-G. Schmidt, Die Relationsfaktorgruppen von Stickelberger-Elementen und Kreiszahlen, J. Reine Angew. Math. 315 (1980), 60-72.

[7] W. Sinnott, On the Stickelberger ideal and the circular units of a cyclotomic field, Ann. of Math. 108 (1978), 107-134.

[8] L. C. Washington, Introduction to Cyclotomic Fields, Springer, New York, 1997.

Dipartimento di Matematica

Via F. Buonarroti, 2

56127 Pisa, Italy

E-mail: dvornic@dm.unipi.it

Received on 1.2.2000

and in revised form on 23.5.2000 